\title{
A Modelagem dos Comportamentos Agressivos e as Ciências Cognitivas
}

\author{
Silvio José Lemos Vasconcellos ${ }^{1}$ \\ Universidade Federal do Rio Grande do Sul \\ Patrícia Picon \\ Gabriel José Chittó Gauer \\ Pontifícia Universidade Católica do Rio Grande do Sul
}

\begin{abstract}
RESUMO - O principal objetivo deste artigo é discutir o modelo de processamento de informações sociais relativo à competência social em crianças e adolescentes. Aventa-se que a reformulação do modelo de processamento das informações sociais (Crick \& Dodge, 1994) apresenta limitações relacionadas ao enfoque conexionista. Os autores não contestam o valor preditivo do modelo, mas postulam que as múltiplas etapas do processamento das informações sociais também podem ser explicadas por intermédio de uma arquitetura híbrida. A principal conclusão é que outra reformulação desse mesmo modelo mostra-se possível. O artigo também discute a dificuldade quanto à obtenção de resultados conclusivos sobre essa questão.
\end{abstract}

Palavras-chave: comportamento agressivo; processamento de informação; ciências cognitivas; conexionismo.

\section{The Modelling of the Aggressive Behaviors and the Cognitive Sciences}

\begin{abstract}
The main purpose of this article is to discuss the social information processing model relative to social competence in children and adolescents. It is assumed that a reformulation of the social information processing model (Crick \& Dodge, 1994) presents limitations related to the connectionist approach. The authors do not contest the predictive value of the model but argue that the multiple steps of the social information processing can also be explained by means of an hybrid architecture. The main conclusion is that another reformulation of this model is possible. This article also discusses the difficulty in obtaining conclusive results regarding this question.
\end{abstract}

Key words: aggressive behavior; information processing; cognitive sciences, connectionism.

O advento das ciências cognitivas como uma nova forma de compreender e explicar o psiquismo humano data do final da década de 1950 (Miller, 2003). Segundo Gardner (2003), o próprio termo teria sido utilizado pela primeira vez em 1956, ao que tudo indica, nos trabalhos de George Miller. Dupuy (1996) ressalta, entretanto, que as origens desse novo paradigma podem estar no próprio fortalecimento da cibernética ocorrido em décadas anteriores. Independente de questões históricas como essas, o fato é que nas últimas décadas, os estudos experimentais sobre as diferentes funções cognitivas têm se mostrado elucidativos para compreender como os seres humanos percebem, aprendem, recordam e ponderam as informações (Sternberg, 2000). Nesse sentido, as ciências cognitivas constituem-se hoje num amplo conjunto de abordagens que procuram investigar e discutir uma série de regularidades que perfazem o funcionamento cognitivo, bem como as decorrentes manifestações comportamentais (Vasconcellos, 2005).

$\mathrm{Na}$ tentativa de elaborar modelos explicativos que mais se aproximem dos aspectos gerais da cognição, surgiram, no âmbito das próprias ciências cognitivas, diferentes tipos de arquiteturas funcionais (Frawley, 2000). Uma arquitetu-

1 Endereço: Av. Protásio Alves 770 / 33, Rio Branco, Porto Alegre, RS, Brasil 90410-004. E-mail: silvv@pop.com.br. ra nada mais é do que um modelo mais amplo que procura abarcar o funcionamento cognitivo, tentando explicar as suas regras ou, de outro modo, o seu próprio dinamismo, considerado, em alguns casos, computacionalmente intratável (Frawley, 2000). Cita-se, como exemplos dessas arquiteturas, o assim denominado representacionalismo, o conexionismo subsimbólico ou mesmo as chamadas arquiteturas híbridas (Frawley, 2000). Na seqüência deste artigo, explicaremos melhor as diferenças entre as arquiteturas referenciadas.

De um modo geral, cabe salientar que o pressuposto maior para que sejam propostas arquiteturas desse tipo relaciona-se à própria discussão sobre o caráter representacional da cognição humana e a suposta adequação de alguns entendimentos computacionais sobre a mesma. Ou seja, se entendermos o substrato neurológico como um hardware, cabe então dizer que é para o funcionamento do software que as ciências cognitivas se voltam (Eysenck \& Keane, 1994). Afinal, conforme salienta Gardner, "qualquer um que quisesse banir o nível representacional do discurso científico, seria obrigado a explicar a linguagem, a solução de problemas, a classificação e coisas semelhantes, estritamente em termos de análise neurológica e cultural" (Gardner, 2003, p. 403).

Neste artigo, discutiremos modelos teóricos que recorrem a algumas das arquiteturas citadas para explicar 


\section{S. J. L. Vasconcellos e cols.}

manifestações de agressividade em crianças e adolescentes. Pode-se dizer, em síntese, que o principal objetivo deste trabalho é tentar elucidar os avanços que os estudos cognitivos têm gerado para a compreensão da etiologia da agressividade e dos comportamentos anti-sociais e, ao mesmo tempo, a dificuldade em se chegar a um modelo geral e único para explicar os aspectos cognitivos subjacentes à manifestação dos comportamentos agressivos.

\section{O modelo de processamento de informações sociais e as suas versões baseadas em arquiteturas cognitivas distintas}

O modelo de processamento de informações sociais proposto por Keneth Dodge buscou, inicialmente, sintetizar o tipo de sequiência que perfaz o processamento das informações sociais em crianças e adolescentes, levando em conta a atuação de capacidades biologicamente determinadas, bem como o repertório de informações presentes e atuantes nesse processo (Dodge, 1986). Em outras palavras, o referido modelo procurou explicitar a forma pela qual as informações vinculadas ao contexto acabam sendo utilizadas nos processos de interação. Deficiências específicas no processamento podem, de acordo com o modelo, subsidiar a adoção de comportamentos agressivos e anti-sociais (Dodge, 1986; Dodge, Bates \& Pettit, 1990; Graham \& Hudley, 1994; Shahinfar, Kupersmidt \& Matza, 2001; Steinberg \& Dodge, 1982).

Várias pesquisas têm respaldado a idéia de que deficiências em etapas específicas do processamento poderiam, realmente, estar relacionadas à manifestação da agressividade em crianças e adolescentes. As pesquisas enfatizaram, por exemplo, o papel dos aspectos sensórioperceptivos que subsidiam a maneira que o sujeito orientase diante das situações. Dodge e Newman (1981) evidenciaram o fato de que crianças com tendências agressivas mostravam-se menos eficazes no tocante à codificação das informações sociais. Conforme esse estudo, as crianças não agressivas do grupo controle demonstraram, em termos quantitativos, uma habilidade maior para perceber e identificar as informações que se fazem presentes num contexto interpessoal.

Perceber, sentir e focalizar a atenção são mecanismos que, significativamente, servem para a consolidação de uma compreensão daquilo que, porventura, pode estar caracterizando uma circunstância específica. Investigações realizadas por Lochman e Dodge (1994) também evidenciaram o fato de que garotos agressivos demonstravam uma menor capacidade de lembrar dos dados relevantes envolvidos numa situação interpessoal de conflito.

De acordo com o modelo, uma segunda etapa diz respeito à interpretação. Pode-se, em termos ilustrativos, destacar os trabalhos de Dodge (1980) e de Dodge e Tolmin (1987), pelos quais ficou caracterizada a tendência de que crianças agressivas tendem a gerar análises menos respaldadas pelos dados percebidos e mais influenciadas pelas experiências anteriores. Ou seja, a própria interpretação que um sujeito com tendências agressivas faz da situação prioriza os dados que podem ser trazidos à memória, em detrimento de outras evidências. De uma forma comple- mentar a esses achados, Bickett, Milich e Brown (1996) e Dodge e Somberg (1987) chamaram a atenção em suas pesquisas para o fato de que crianças e adolescentes agressivos atribuem mais hostilidade quanto às intenções alheias do que crianças e adolescentes não agressivos. Dodge, Murphy e Buchsbaum (1984) também demonstraram que um evento ambíguo, como, por exemplo, ser atingido por trás por uma bola enquanto a criança passeia numa praça, tende a ser interpretado, por uma criança agressiva, como intencional.

Uma vez interpretada a situação, o modelo de processamento das informações sociais estabelece que o sujeito irá buscar respostas condizentes com a situação que acabou de ser contemplada. Dessa forma, ocorre um processo gerativo no tocante às diferentes atitudes que supostamente mostram-se adequadas ao contexto. Antes da tomada de decisão são verificadas as possíveis alternativas a serem adotadas. A esse respeito, as pesquisas desenvolvidas por Lochman e Dodge (1994), bem como por Mize e Cox (1990) oferecem evidências sobre o fato de que crianças e adolescentes agressivos acabam gerando um número menor de possíveis soluções diante de uma determinada situação de impasse. Por outro lado, também ficou evidenciado que crianças e adolescentes passíveis de serem caracterizados como agressivos tendem a confiar mais na própria eficácia das estratégias agressivas, sendo que tais estratégias costumam ser referenciadas antes de quaisquer outras soluções no que diz respeito a uma situação hipotética de impasse (Richard \& Dodge, 1982).

Uma outra fase diz respeito ao processo de decisão. Nessa fase, os sujeitos irão considerar a própria funcionalidade das respostas geradas, sendo que a escolha vinculase ao êxito ou ao fracasso em situações anteriores, além de estar atrelada à imagem que a criança e o adolescente têm de si mesmo. Crick e Dodge (1994) demonstraram que um subgrupo de crianças agressivas, aquelas que tendem a utilizar o comportamento agressivo de forma mais pró-ativa, se sentem mais eficazes no emprego da agressão quando comparada aos seus pares. Uma outra pesquisa também evidenciou que crianças e adolescentes agressivos esperam, em geral, menos resultados positivos quando consideram a possibilidade de se valerem de atitudes pró-sociais em situações hipotéticas de impasse (Quiggle, Garber, Panak \& Dodge, 1992).

Ainda no tocante a esse modelo de processamento das informações sociais, ressalta-se o papel dos processos de atuação que implicam em uma auto-regulação comportamental. Dito de outro modo, uma vez que as ações humanas podem manifestar-se de forma roteirizada, as estratégias escolhidas envolvem também a utilização de outras informações referentes ao transcurso da ação. O sujeito acaba, portanto, servindo-se de informações relevantes que estão armazenadas na memória de longo prazo, para monitorar as conseqüências supostamente previsíveis de seus atos, ou mesmo as vicissitudes que irão caracterizar a circunstância de interação social (Crick \& Dodge, 1994).

Percebe-se, por intermédio desses estudos, que o modelo de processamento de informações sociais trata de considerar as diferentes etapas que podem estar envolvidas nos processos de interação social, bem como as próprias 
funções cognitivas que lhe dão suporte. O modelo reformulado proposto por Crick e Dodge em 1994, diferencia-se, entretanto, do modelo anteriormente proposto por Dodge (1986). Para os autores, já não mais seria possível explicar as etapas que perfazem o modelo com base numa concepção de processamento seqüencial (Crick \& Dodge, 1994). Conforme as palavras dos próprios autores, o modelo reformulado passou a ser "uma tentativa de caracterizar as atividades cerebrais no campo da cognição, em termos de numerosos sistemas computacionais paralelos (redes neurais) em um nível que está abaixo da linguagem simbólica" (Crick \& Dodge, 1994, p. 77). Portanto, mais do que enfatizar as etapas de processamento, os autores passaram a concebê-las como ocorrendo em um nível que não pode ser devidamente abarcado por um processamento meramente seqüencial de informação.

O tipo de arquitetura cognitiva, utilizada para essa nova proposta, está fundamentada numa espécie de produto tensor da rede como um todo (Smolensky, 1990) e não em regras que supostamente controlam o processamento. Nesse sentido, a ênfase recai mais no dinamismo do que nos algoritmos executados. Se o antigo modelo enfatizava que as etapas de processamento ocorriam de um modo plenamente seqüencial, a sua respectiva reformulação tratou de chamar atenção para os processos de coativação que podem estar retroalimentando ou restringindo cada uma dessas etapas. Pode-se afirmar, ainda, que essa mesma reformulação exemplifica algumas das diferenças entre uma arquitetura de processamento seqüencial de informação que pode, segundo Frawley (2000), ser denominada como representacionalista e o assim chamado conexionismo subsimbólico. Pode-se afirmar também que essa nova proposta de modelagem está de acordo com um dos grandes méritos do conexionismo destacado por Teixeira (1998), ou seja, mostrar que certos estados mentais ocorrem em algum lugar da rede, embora não saibamos onde. $\mathrm{Na}$ seqüência, discutiremos, entretanto, outra possibilidade interpretativa relacionada à adoção desse paradigma como forma de explicar os aspectos cognitivos que podem ser subjacentes à manifestação de comportamentos agressivos e anti-sociais.

\section{Aspectos gerais da modelagem}

Diante das próprias pesquisas citadas, parece pouco contestável o fato de que o processamento de informações sociais em questão demonstra englobar etapas que, por sua vez, relacionam-se diretamente à manifestação de comportamentos agressivos em crianças e adolescentes. As evidências corroboram a premissa de que certas deficiências no processamento contribuem para um maior nível de agressividade gerada em situações de interação social (Dodge, 2003). O modelo não se apresenta, entretanto, como uma tentativa de elucidar todos os aspectos etiológicos relacionados à manifestação de comportamentos agressivos. Não há, por parte dos autores, a pretensão de explicar a questão da agressividade na infância e na adolescência, contemplando todos os seus níveis. Conforme destaca Dodge, trata-se principalmente da "descrição da fenomenologia da interação social.” (1993, p. 563).
Ao descrever essa fenomenologia, Crick e Dodge (1994) recorrem, conforme já salientado, ao conexionismo subsimbólico. A idéia de um processamento de informações sociais, dentro do qual deficiências específicas subsidiam comportamentos específicos, demonstra ser apropriada. Mas será que o conexionismo subsimbólico é a única forma capaz de fundamentar uma modelagem quanto a esse processamento?

Crick e Dodge (1994) propuseram que, sobre cada etapa de processamento, poderia ocorrer uma ação direta dos esquemas sociais, sendo que as etapas poderiam agir umas sobre as outras ou ainda sobre os próprios esquemas. Com base na idéia de que um esquema refere-se à estrutura que viabiliza a organização do conhecimento, postulou-se que etapas de processamento são, na verdade, o resultado das diferentes forças que operam na rede como um todo (Crick \& Dodge, 1994). Esse é um dos pressupostos centrais do conexionismo, segundo o qual um atrator, que pode, por exemplo, vincular-se a uma informação contextual, seleciona um subconjunto de operações dentro do conjunto de operações possíveis no sistema (Varela, 1994). A consolidação de uma atribuição de hostilidade diante de uma situação de impasse não seria uma operação inserida numa sequiência de outras operações como postulava o modelo anterior, mas sim um padrão de ativação regido pelas forças de neuroconectividade. O conexionismo subsimbólico dispensa, dessa forma, a noção de que determinadas operações cognitivas sejam regidas por algoritmos específicos (Varela, 1994). O sistema aprende e funciona com base naquilo que ora se torna ativo, ora se torna inativo dentro da rede. Conforme salienta Pinker (1999), o conexionismo é também um tipo de computacionalismo no qual predomina uma estatística multivariada.

A questão, entretanto, é que o cálculo tensorial pode não ser a única explicação possível para a consolidação das próprias etapas de processamento. Uma regra de produção do tipo "se...então..." pode igualmente explicar a consolidação do citado mecanismo. Numa situação na qual, conforme o estudo de Dodge e cols. (1984), uma criança é atingida por trás com uma bola e interpreta o referido ato como sendo intencional, pode ter ocorrido tão somente a execução de um algoritmo na fase de interpretação. Ou seja, para a criança: "se algo me atinge numa situação onde nenhuma outra informação contextual pode ser avaliada, então infiro que foi um ato proposital". Por outro lado, a regra de produção refere-se, nesse caso, à execução de uma interpretação condicional na qual não há a necessidade da recuperação de um conhecimento declarativo sobre a informação (Anderson, 1976). Cabe, entretanto, ressaltar alguns pontos sobre as questões que estão por trás dessa afirmação.

Em primeiro lugar, a idéia de que, numa interpretação desse tipo, poderia ter ocorrido a execução de um algoritmo prescinde de uma discussão sobre como o próprio hardware implementa a regra em questão. Além disso, o atual estágio de conhecimento sobre o assunto não permite explicações conclusivas a esse respeito. Por outro lado, tal como admite Smolensky (1998), essa mesma discussão acaba também ficando em aberto no que se refere ao próprio conexionismo subsimbólico. O que 


\section{S. J. L. Vasconcellos e cols.}

está sendo discutido aqui, no entanto, é se, por exemplo, interpretar o ato de ser atingido por trás com uma bola é exclusivamente explicado como sendo o subproduto de tensões na rede ou se, de outro modo, pode também ser explicado como uma "regra dura" para utilizar as próprias palavras de Smolensky (1998). Aventamos, neste artigo, que a interpretação pode consolidar-se a partir de uma regra dura, embora o processamento como um todo não seja passível de uma explicação algorítmica como iremos explanar na seqüência.

Um segundo ponto que merece ser elucidado a respeito da modelagem alternativa que estamos propondo é o fato de que postular uma operação do tipo: "se algo me atinge numa situação onde nenhuma outra informação contextual pode ser avaliada, então infiro que foi um ato proposital" não é, evidentemente, postular um ato deliberado por parte da criança. Tal pressuposto está de acordo com o conceito de regra de produção enfatizado anteriormente. Trata-se tão somente de entender que determinadas regras de inferência contribuem para a manifestação de comportamentos agressivos. Nesse sentido, as regras que a criança adquire ao longo do seu desenvolvimento acabam por ser executadas e fundamentam as etapas do processamento. Enfatizamos, dessa forma, que qualquer modelagem aproximativa do modo como crianças e adolescentes processam as informações sociais e da sua respectiva relação com a agressividade pode considerar a ocorrência dessas regras. A partir disso, apresentamos uma proposta alternativa para essa mesma modelagem.

\section{A pertinência de uma arquitetura híbrida para a ela- boração de um modelo alternativo}

Uma arquitetura de inclusão nada mais é do que uma forma de explicar diferentes aspectos do funcionamento cognitivo no qual as redes conexionistas explicam os principais aspectos desse funcionamento, sem excluir a possibilidade de certas regras e regularidades operem no sistema (Dupuy, 1996).

Uma das críticas mais contundentes feitas por Fodor e Pylyshyn ao conexionismo é o fato de que modelagens desse tipo demonstram uma certa inaptidão para explicar as propriedades composicionais do pensamento (Fodor \& Pylyshyn, 1988). Pinker (1999) afirma que o pensamento precisa de certas regras estáveis para mostrar-se recursivo. Hinton (1992) assinala que o conexionismo precisaria de algum tipo de sistema de instrução para consolidar a sua própria funcionalidade.

A acepção que estamos defendendo refere-se ao fato de que a modelagem do processamento de informações sociais em crianças e adolescentes pode abarcar certas regras. Em contrapartida, tal afirmação não se coaduna com a idéia de que o funcionamento cognitivo como um todo resulte tão somente da execução de regras identificáveis. Postular uma arquitetura de inclusão para a modelagem em questão equivale a afirmar que, dentro do sistema, podem existir microrrepresentações, com caráter estável e, conforme destaca Andler (1998), funcionando a partir de regras computacionais. Nesse sentido, procuramos, no presente artigo, conciliar as citadas interpretações com o próprio modelo de informações sociais que está sendo discutido.

Com base nas forças que se tornam mais ou menos ativas na rede, certas regras de codificação, de interpretação, de geração de respostas e de decisão são ou não acionadas. As regras podem ser "duras" pois precisam ser recursivas para o sujeito que se encontra em uma situação de interação social. Por outro lado, o modo como tais regras são alocadas é dinâmico e vulnerável aos diferentes processos de coativação. Essa arquitetura continua estando em concordância com o mérito da reformulação proposta por Crick e Dodge (1994), ou seja, abarcar um processamento on-line sustentado por estruturas mentais latentes.

O conhecimento social, nessa concepção, não está armazenado em esquemas fixos na memória e esse entendimento está, por sua vez, de acordo com o próprio conexionismo subsimbólico, sem recorrer ao conexionismo ultralocalista criticado por Smolensky (1998). De outro modo, os processos cognitivos que exigem um maior nível de especificidade e estabilidade poderiam ser regidos por regras. De acordo com esse entendimento, as regras não seriam necessariamente selecionadas por outras regras. A informação contextual que se mostra capaz de especificar subconjuntos dentro dos conjuntos possíveis, também seria capaz de acionar regras específicas. Faz-se aqui uma analogia com modelos advindos dos estudos sobre inteligência artificial, baseados, por sua vez, em arquiteturas híbridas por intermédio das quais os planos de ação podem ser acionados tanto por metas previamente estabelecidas, pela regularidade das informações contextuais ou por uma reatividade diante de alterações súbitas no contexto (Georgeff, 1987).

Essa idéia pode ser melhor explicada com a afirmação de que não seria verdadeiramente possível saber, por exemplo, quando uma criança ou adolescente, no transcurso do processamento, pode consolidar a interpretação. Em contrapartida, seria possível saber que o seu padrão interpretativo, quando acionado, pode envolver regras específicas. Afirmamos, portanto, que esse caráter irregular e, ao mesmo tempo, regular do processamento das informações sociais pode também ser explicado por uma modelagem baseada em uma arquitetura híbrida. Dito de outra forma, pode estar fundamentado em uma arquitetura que contemple tanto um processamento massivamente distribuído em paralelo, como também, certos algoritmos que melhor explicam o caráter repetitivo de alguns mecanismos envolvidos.

\section{Considerações Finais}

Quando Crick e Dodge (1994) propuseram uma reformulação do modelo de processamento das informações sociais, objetivaram explicar, de forma mais apropriada, os aspectos mais dinâmicos desse mesmo processamento. Neste artigo, estamos sugerindo a viabilidade de um outro modelo compatível com as etapas de processamento salientadas anteriormente. Essa proposta vincula-se ao fato de que tais etapas também podem ser explicadas pela execução de certos algoritmos e pela sua respectiva 
coexistência com o dinamismo característico dos modelos conexionistas.

Entendemos, contudo, que explicar como o cérebro implementa esse "programa" não é uma tarefa fácil. Por outro lado, essa proposta é coerente com o cognitivismo, uma vez que, em última instância, essa abordagem interdisciplinar sustenta que é coerente adotar uma ontologia não substancialista e, ao mesmo tempo, não reducionista (Dupuy, 1996).

A proposta apresentada neste trabalho foi, entretanto, mais específica. Procuramos assinalar novas possibilidades interpretativas diante do modelo de processamento das informações sociais proposto por Crick e Dodge (1994). Postulamos que abandonar o pressuposto de que certas regras possam consolidar as etapas de codificação, interpretação e geração de resposta é um empreendimento capaz de acrescentar um nível de complexidade desnecessária quanto à própria modelagem. Essa complexidade que, por sua vez, baseia-se num amplo e irrestrito dinamismo no sistema, demonstra não ser a única modelagem plausível, uma vez que existe a própria necessidade de explicar o caráter estável e recorrente de certos mecanismos.

De um modo geral, este artigo procurou sugerir um modelo alternativo e, até certo ponto, complementar ao modelo de processamento das informações sociais de Crick e Dodge (1994). Não apresentamos, entretanto, nenhum tipo de contestação quanto à enorme pertinência do modelo em questão para explicar como certos aspectos da cognição social operam e contribuem para a manifestação de comportamentos agressivos em crianças e adolescentes. O pressuposto de que certas deficiências em etapas do processamento podem contribuir para tais comportamentos demonstra estar bastante respaldado por uma série de pesquisas empíricas. A questão que se coloca é sobre outras formas de explicar esse processamento no que se refere às suas propriedades ou, poder-se-ia dizer, ao seu modo de ocorrência como um fenômeno mental irredutível. Essa é uma discussão que merece, no entanto, novas e abrangentes considerações.

\section{Referências}

Anderson, J. R. (1976). Language, Memory and Thought. Hilsdalle: Erlbaum.

Andler, D. (1998). Cálculo e representação: as fontes Em D. Andler (Org.), Introdução às Ciências Cognitivas (pp. 25-54). São Leopoldo: Editora Unisinos.

Bickett, L. R., Milich, R. \& Brown, R. T. (1996). Attributional styles of aggressive boys and their mothers. Journal of Abnormal Child Psychology, 24, 457-472.

Crick, N. R. \& Dodge, K. A. (1994). A Review and Reformulation of Social Information-Processing Mechanisms in Children's Social Adjustment. Psychological Bulletin, 115, 74-101.

Dodge, K. A. (1980). A. Social cognition and children's aggressive behavior. Child Development, 51, 162-170.

Dodge, K. A. (1986). A social information processing model of social competence in children. Em M. Perlmutter (Org.), Minnesota symposia on child psychology: Vol. 18. Cognitive perspectives in children's social and behavioral development. Hillsdale: Erlbaum.
Dodge, K. A. (1993). Social-cognitive mechanisms in the development of conduct disorder and depression. Annual Review of Psychology, 44, 559-584.

Dodge, K. A. (2003). A Biopsychosocial Model of the Development of Chronic Conduct Problems in Adolescence. Developmental Psychology, 39, 349-371.

Dodge, K. A., Bates, J. E. \& Pettit, G. S. (1990). Mechanisms in the cycle of violence. Science, 250, 1678-1683.

Dodge, K. A., Murphy, R. M. \& Buchsbaum, K. (1984). The assessment of intention-cue discrimination cues in children: Implications for developmental psychopathology. Child Development, 55, 163-173.

Dodge, K. A. \& Newman, J. P. (1981). Biased decision making processes in aggressive boys. Journal of Abnormal Psychology, 90, 375-379.

Dodge, K. A. \& Somberg B. R. (1987). Hostile Attributional biases among aggressive boys are exacerbated under conditions of threats to the self. Child Development, 58, 213-224.

Dodge, K. A. \& Tomlin, A. M. (1987). Utilization of self-schemas as a mechanism of interpretational bias in aggressive children. Social Cognition, 5, 280-300.

Dupuy, J. (1996). Nas origens da ciência cognitiva. São Paulo: Unesp.

Eysenck, M. W. \& Keane, M. T. (1994). Psicologia Cognitiva: um manual introdutório. Porto Alegre: Artmed.

Fodor, Z. W. \& Pylyshyn J. (1988). Connectionism and cognitive architecture: A critical analysysis. Cognition, 28, 3-17.

Frawley, W. (2000). Vygotsky e a Ciência Cognitiva - Linguagem e integração das mentes social e computacional. Porto Alegre: Artmed.

Gardner, H. (2003). A nova ciência da mente. São Paulo: Edusp.

Georgeff, M. P. (1987) Planning. Annual Review of Computer Science, 2, 359-400.

Graham, S. \& Hudley, C. (1994). Attributions of aggressive and nonagressive African-American male early adolescents: A study of construct accessibility. Development Psychology, 30, 365-373.

Hinton, G. E. (1992). How Neural Networks Learn From Experience. Scientific American, 267, 3.

Lochman E. J. \& Dodge K. A. (1994). Social Cognitive Processes of Severely Violent, Moderately Aggressive and Nonagressive Boys. Journal of Consulting and Clinical Psychology, 62, 366-374.

Miller, G. A. (2003). The cognitive revolution: a historical perspective. Trends in Cognitive Sciences, 7, 141-144.

Mize, J. \& Cox, R. (1990). Social Knowledge and social competence: Number and quality of strategies as predictors of peer behavior. The Journal of Genetic Psychology, 151, 117-127.

Pinker, S. (1999). Como a mente funciona. São Paulo: Companhia das Letras.

Quiggle, N., Garber, J., Panak, W. \& Dodge, K. A. (1992). SocialInformation processing in aggressive and depressive children. Child Development, 63, 1305-1320.

Richard, B. A. \& Dodge, K. A. (1982). Social maladjustment and problem solving in school-aged children. Journal of Consulting and Clinical Psychology, 50, 226-233.

Shahinfar, A., Kupersmidt, J. B. \& Matza, L. S. (2001). The relation between exposure to violence and social information processing among incarcerated adolescents. Journal of Abnormal Psychology, 110, 136-141. 


\section{S. J. L. Vasconcellos e cols.}

Smolensky, P. (1998). IA conexionista, IA simbólica e cérebro. Em D. Andler. (Org.), Introdução às Ciências Cognitivas (pp. 77-100). São Leopoldo: Editora Unisinos.

Smolensky, P. (1990). Tensor product variable binding and the representation of symbolic structures in connectionist network. Artificial Intelligence, 46, 159-216.

Steinberg, M. S. \& Dodge, K. A. (1982). Attributional bias in aggressive adolescents boys and girls. Journal of Social and Clinical Psychology, 1, 312-321.

Sternberg, R. J. (2000). Psicologia Cognitiva. Porto Alegre: Artmed.

Teixeira, J. F. (1998). Mentes e máquinas: Uma introdução à ciência cognitiva. Porto Alegre: Artes Médicas.
Varela, F. (1994). Conhecer - As ciências cognitivas: Tendências e perspectivas. Lisboa: Instituto Piaget.

Vasconcellos, S. J. L. (2005). A Mente entreaberta: Reflexões sobre o que a psicologia científica anda pensando sobre o nosso pensar. Rio de Janeiro: Ciência Moderna.

Recebido em 18.04.2005

Primeira decisão editorial em 24.06.2005

Versão final em 02.05.2006

Aceito em 30.05.2006 